\title{
Oral Prosthetic Rehabilitation for Alzheimer Patients: Planning, Procedures, and Follow-Up
}

\section{Tânia e Silva Pulicano Lacerda*}

Department of Odonthology, City University of São Paulo, São Paulo, Brazil

\begin{abstract}
This work aims at discussing prosthetic oral rehabilitation for Alzheimer patients, from planning until followup. In general, techniques for making dentures and implants for such patient are the same used in a healthy one. However, Alzheimer's Disease does not affect only patients, but also their entire personal relations, such as family members, caregivers, and, in a broader sense, all of society. Throughout this paper, a session on the importance of accessibility in dental clinics for these patients will be developed, followed by the peculiarities in planning their dental prosthesis, as well as orientations on diets, hygiene, and mastication. Thus, the planning of treatments will be altered according to the general physical state of the patient, his or her degree of dependence on others, and life expectancy. In later stages of the disease, for instance, the patient is commonly affected by dysphagia, which the dentists while dealing with Alzheimer patients are innumerous, which emphasizes the need for the dentist to maintain an inter professional line of communication, for example, with physicians, nutritionists, and speech therapists, providing the patient with a singular treatment plan. decisions. The challenges
\end{abstract}

\section{Introduction: The Brazilian Context and Patients' Profiles}

Reflecting upon the prosthetic rehabilitation for Alzheimer patients, it is important to keep in mind that such individual presents certain singularities, which must be recognized since the very beginning. Thus, the objective of this paper is to call on professionals' attention to the specificities of all stages of treatment: planning, executing, and the necessary follow-up measures. The major distinction is the interprofessional approach in the management of each case, which includes the work of physicians, speech therapists (fonoaudiologists), and nutritionists. While examining patients as subjects with the complexities of any individual, physical, emotional, and spiritual aspects are all involved, and we cannot overlook any of these elements.

Alzheimer's disease does not affect only patients, but also their entire personal relations, such as family members, caregivers, and, in a broader sense, all of society. In dentistry, at least at this moment in Brazil, professionals seldom have adequate background to attend the specific needs of Alzheimer patients. Geriodontics was only recognized by the Conselho Federal de Odontologia as a specialty in 2002. In order to give you a better idea of what this represents, in 2013, Brazilian population was approximately 201 million.

Senior population reached 23,5 million (which means $10.8 \%$ of the total), while there were only 283 specialists, distributed mostly in the Southern, Southeastern and coastal regions of the country.

\section{Accessibility in Dental clinics for Patients with Alzheimer's Disease}

Unlike countries where there are more regulated laws for accessibility, this process is still ongoing in Brazil. The clinic needs to be adequate to receive these specific patients in the different stages of the disease, also being prepared for home care sessions with proper tools and equipments. It is important to remember that patients can become dependent on the wheel chair, and the clinic must be adapted to them. The process of placing the prosthesis can be made on the dentist's chair, on the wheel chair and, in other situations, on the hospital bed. While planning the prosthetic work, the dentist must also take into consideration the number of visits to clinic that will require someone to accompany the patient.

\section{Peculiarities in the Planning of Dental Prosthesis for Alzheimer Patients}

In order to fulfill these patients' needs, the dentist has to be very careful at the preparation of the staff. From the first minute of scheduling the appointment, the dentist's assistants must ensure all matters at the patient's convenience, like trajectory, time and date of the visit.

In other words, the staff needs to be prepared to identify the need to talk to others since the very beginning. In the case of evaluating Alzheimer patients, one needs to determine the degree of functionality and dependence.

When patients get to the clinic, besides observing the aspects of physical dependence, analysis of their cognitive skills is highly productive. Sometimes, prior to the prosthetic treatment, patients are already dealing with Alzheimer's, other times, they start presenting lapses or signs that may indicate the development of the disease.

One of the most common standard preliminary tests is the Mini Mental State Examination (MMSE), whose scoring system has been adapted to the Brazilian context, given the differences in literacy rates. Analyzing the results, the dentist is able to direct the patient to a specialized physician.

From the beginning of the treatment, a speech therapist's evaluation is indicated in order to discuss the planning of the oral rehabilitation. Such professionals may work at the beginning, during, or after the execution of the prosthesis.

It is necessary to identify, in this process, if the person presents signs of dysphagia, which can importantly compromise the planning of

*Corresponding author: Tânia e Silva Pulicano Lacerda, Department of Odonthology, City University of São Paulo, São Paulo, Brazil, Tel: 1-650-268-9744; Fax: 1-650-6181414; E-mail: tanialacerda@col.odo.br

Received October 07, 2015; Accepted October 29, 2015; Published November 05, 2015

Citation: Lacerda TSP (2015) Oral Prosthetic Rehabilitation for Alzheimer Patients: Planning, Procedures, and Follow-Up. J Gerontol Geriatr Res 4: 251. doi:10.4172/2167-7182.1000251

Copyright: (c) 2015 Lacerda TSP. This is an open-access article distributed under the terms of the Creative Commons Attribution License, which permits unrestricted use, distribution, and reproduction in any medium, provided the original author and source are credited. 
the prosthetic treatment. A survey on dysphagic alterations, in cases of dementia, demonstrated: weight loss, dehydration, malnutrition, signs of penetration and/or tracheal suctioning, as well as pneumonia, what, on its turn, can lead to death. When the patient presents dysphagia, the dentist, the speech therapist, and the nutritionist must establish together a singular therapeutic project, in order to define any changes in alimentation, set meal times, and alter the consistency of the foods. In some cases, the lower removable prosthesis, even helping mastication, may interfere in the swallowing process and then, remotion, or readaptation, after the myofunctional work, is indicated. Sometimes, not so frequently, both prosthesis (upper and lower) need to be removed. The speech therapist may also need to request further tests to determine the direction of the therapeutic process, and/or the diagnosis of dysphagia, for instance, the video deglutogram. In advanced stages, it is important to remember that patients might benefit from an alternative way of eating, for example, nasogastric tubes orgastrostomy, no longer needing their mouth and teeth.

In technical terms, there are no differences between making a prosthesis for a young or senior person. The differences start to surface while dealing with external factors: on one hand, there may be other pathologies, such as Parkinson or Diabetes, which have to be properly addressed with the physician. On the other, there may be difficulties related to the patient's social environment, family support, caregivers, and financial access to services.

In the moment of the execution, the dentist must be aware of the medications taken by the patient in order to know whether any of them will have side effects that might harm the making or retention of the prosthesis, such as involuntary muscular movements and hypo salivation.

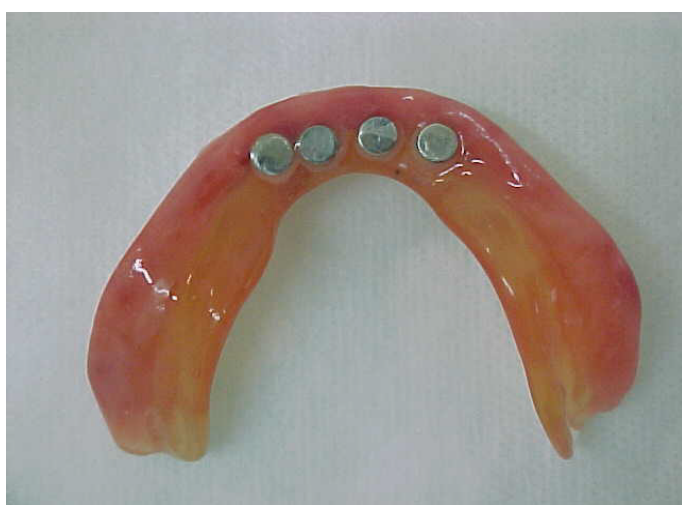

Figure1: Overdenture with magnets

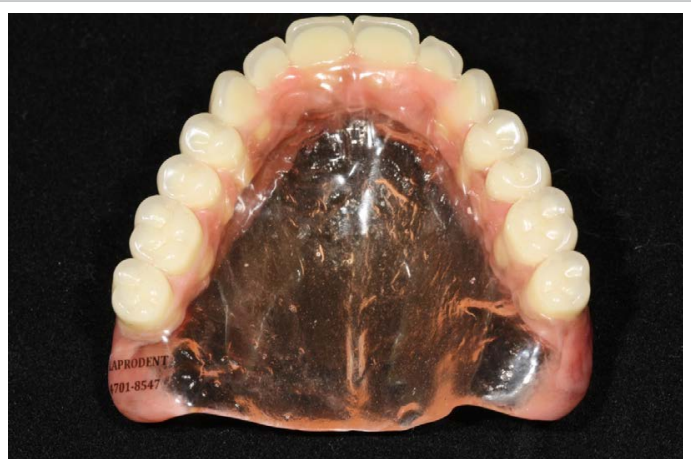

Figure 2: Complete denture labeled with patient's personal contact information.
Subsequently, during the treatment, it is important to guarantee that the patient is properly seated on the chair, that the impression materials are correctly handled, and that the surgeon and the staff are skilful to prevent the material from leaking towards the throat, especially in cases of dysphagia.

When the patient uses removable prosthesis, it is easier to clean them, while fixed ones demand more motor skills, or the caregiver's manual abilities. Magnets (Figure 1) have proved to be a good solution for patients who already have lower implants and for those with residual roots, once the overdentures present better retention than conventional prosthesis.

The magnetic action is more helpful in the process of placing the prosthesis comparing to other forms of retention, such as bars and o'rings. However, if the patient is a pacemaker carrier, magnets are not indicated. It is important to highlight that adhesives can help in the stabilization of well-adapted prosthesis.

When removable prosthesis are chosen, in the processing phase of the denture bases it is recommended to print the contact information of patients, once they may lose the prosthesis in their daily routine, at home, at their long term care institutions, and even in the hospital.

Aware of the stage of the disease, the dentist must take into consideration the possibility of alternative, less invasive treatments. The professional must focus on dealing with infections, inflammations, and pain. Furthermore, the need for the making of a new prosthesis, the re-adaptation of former ones (rebasing, adjusting), or even the nonexecution of the prosthesis, followed by palliative care of the remaining teeth, when present, have to be discussed.

The explanation of the diagnosis plans of treatment, and instructions for the patient must be objective and clear. If patients have to make a decision, it is important to allow them proper time to process their thoughts. Depending on the stage of the disease, written instructions are recommended, as well as a good communication with a family member and caregivers.

While selecting the best kind of prosthesis for the patient, the dentist should remember the difficulties for hygiene that these patients will probably face throughout the development of the disease, due to the impairment of their cognitive and motor skills, especially towards more advanced stages. At first, patients are autonomous, but with the gradual loss of their independence, a third party will probably need to do it for them. Because of that, removable prosthesis are easier than fixed ones. On the other hand, patients may lose them, or even accidentally throw them away, so a recommendation to avoid these issues is to identify the patient's prosthesis, labeling them with name and telephone number while processing the bases. This identification must be placed preferably in the posterior part of the denture, in a less visible area, such as the vestibular one, on the distal of the last molar (Figure 2). Another interesting alternative is the confection of a clone, which can help in cases of hospitalization, where patients most commonly lose their dentures, as well as when the old prosthesis need to be rebased.

\section{Adjustments of the Prosthesis and Follow-Up: Orientations on Diets, Hygiene, and Mastication}

Similar to the process of making the prosthesis, the maintenance of patients can be done in various places, according to each case. Commonly, adjustments of the prosthesis are done, and hygiene, diet, and mastication orientations are provided. When thinking about oral hygiene for seniors, we must keep in mind: Can the patient do it by him or herself? If so, can they go to the bathroom or will they do it in bed? 


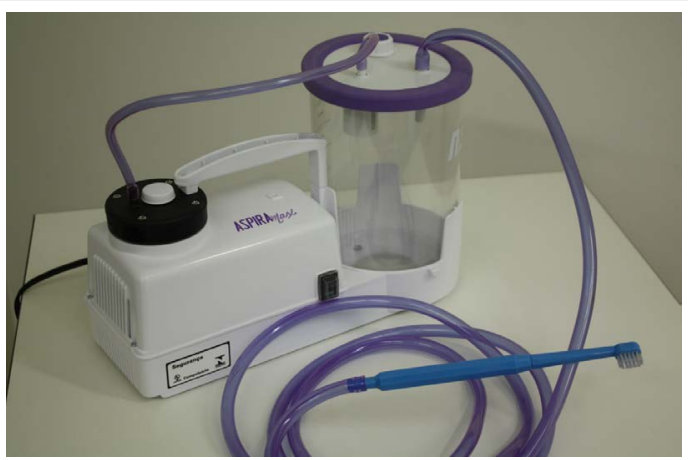

Figure 3: Toothbrush with electric surgical aspirator.

It is important that patients are filled with daily activities that will allow them to have a routine, especially for those with restricted mobility. So, even though the focus here is Alzheimer patients, we cannot forget to think about their cognitive and motor skills, and give special assistance when needed.

Some quite simple and practical measures can be applied. For example, making sure they have access to a mirror, because, if they are able to follow commands, instructions may be written down and attached to it.

The instructions, types of toothbrush, and material will depend on the style of the prosthesis the patients have. However, special needs are required when patients in more advanced stages of the disease present dysphagia. Depending on the caregiver's ability, the dentist may recommend little amounts of toothpaste with a toothbrush and electric surgical aspirator (Figure 3), and, at other times, the dentist may suggest not using toothpaste at all. In this situation, in order to maintain the taste and refreshing sensation of the oral hygiene, a mint tea with a thickener can be given to the patient.
The interprofessional work is once again surfaced in this phase. The nutritionist and speech therapist are constantly providing assistance in terms of the alimentation and proper diet to guarantee the success of the treatment.

\section{Alimentation Changes Throughout the Development of Alzheimer's Disease}

It is indicated, after the installation of the complete dentures, an adaptation to the patients' diets, at least during the first weeks. A liquid-pasty diet is recommended and the consistency of the foods needs to be altered as the patient is feeling more comfortable with the new prosthesis. For Alzheimer patients in more advanced stages of the disease, the diet shall remain liquid-pasty, or even evolve into a parenteral method of feeding (gastrostomy), as previously stated, and according to the evaluation of both the speech therapist and the nutritionist. An excessive soft diet may not contain all the adequate nutrients for the patients' biological needs, generating anemic and apathetic states, which, in more susceptible patients, can lead to death.

The interprofessional work is very important in this phase. Even though the dentist is capable to make general nutritional recommendations, only the nutritionist can prescribe personalized menus, appropriate for each stage of the disease, taking into account the prosthesis' time of use, identifying the need of complementing the alimentation. Once again, the evaluation of the muscles involved in the process of mastication asks for the support of a speech therapist, who can guarantee a better efficacy and alimentary dynamic.

\section{Final Remarks}

The challenges while treating an Alzheimer patient are innumerous, which emphasize the need for the dentist to maintain an interprofessional line of communication, alongside with family members and caregivers. This is an enabling moment for the facing of the many variables each case may present. 